\title{
España y Rusia: Factores Inciertos para hacer negocios*1
}

\section{Spain and Russia: Uncertain Factors in Doing Business}

\author{
Isadora Sánchez-Torné * \\ Macarena Pérez-Suárez ${ }^{* *}$ \\ Juan Carlos Morán-Álvarez ${ }^{* * *}$
}

\section{RESUMEN}

La creación de empresas ha adquirido gran notoriedad en los últimos diez años como instrumento territorial para contrarrestar las dominantes tasas de desempleo. De ahí que se trate de una de las intervenciones más decididas entre 1998-2018 por parte de los gobiernos, que dirigen importantes esfuerzos a promover y dirigir el emprendimiento. La actividad empresarial incide directamente en el crecimiento económico de un país, por lo que requiere intervenir sobre los factores inciertos para iniciar un negocio que representan límites de productividad y competitividad empresarial. En consecuencia, el objetivo de esta investigación fue determinar los

1 Artículo de Reflexión cuyo origen reside en la curiosidad por la economía del país de Rusia a partir de iniciar una colaboración profesional entre el Grupo de Investigación de Planificación y Análisis Económico de la Universidad de Sevilla y una entidad académica de Moscú.

(*) Economista, Universidad de Sevilla, Sevilla, España. Magister en Estudios Avanzados en Dirección de Empresas. Profesora del Departamento de Economía Aplicada III e Investigadora el Grupo de Investigación de Planificación y Análisis Económico de la Universidad de Sevilla. Facultad de Ciencias Económicas y Empresariales. Avda. Ramón y Cajal, 1. C.P: 41018, Sevilla, España. E-mail: isanchez6@us.es

$\left({ }^{* *}\right)$ Doctora en la Universidad de Sevilla, Sevilla, España. Magister en Gestión y Desarrollo de Recursos Humanos. Profesora del Departamento de Economía Aplicada III e Investigadora el Grupo de Investigación de Planificación y Análisis Económico de la Universidad de Sevilla. Facultad de Ciencias Económicas y Empresariales. Avda. Ramón y Cajal, 1. C.P: 41018, Sevilla, España. E-mail: mperez32@us.es

$\left({ }^{* *}\right)$ Economista, Universidad de Sevilla, Sevilla, España. Doctor en Ciencias Económicas. Profesor Titular de Universidad en el Departamento de Economía Aplicada III e Investigador del Grupo de Investigación de Planificación y Análisis Económico de la Universidad de Sevilla. Facultad de Ciencias Económicas y Empresariales. Avda. Ramón y Cajal, 1. C.P: 41018, Sevilla, España. E-mail: jcmoran@us.es

(C) Los autores. Este artículo es publicado por Pensamiento Crítico de la Facultad de Ciencias Económicas, Universidad Nacional Mayor de San Marcos. Este es un artículo de acceso abierto, distribuido bajo los términos de la licencia Creative Commons Atribucion - No Comercia_Compartir Igual 4.0 Internacional. (http://creativecommons.org/licenses/by-nc-sa/4.0/) que permite el uso no comercial, distribución y reproducción en cualquier medio, siempre que la obra original sea debidamente citada. 
factores más problemáticos detectados por los directivos de las empresas relevantes en la economía de Rusia (centralizada) y de España (descentralizada). Para ello, se realizó un análisis estadístico descriptivo y comparativo a partir de la encuesta anual del Wold Economic Forum durante el periodo 2011-2016. Las principales averiguaciones señalaron que los factores más problemáticos para España en relación a Rusia fueron cinco, entre los que destacan la Ineficiente Burocracia Gubernamental y las Tasas de Impuestos. En contraposición, los factores más problemáticos para Rusia en relación a España fueron, sobre todo, la Regulación Fiscal y la Inflación.

Palabras clave: Desarrollo económico; economía internacional; política económica; empresa; factores de riesgo.

JEL: F10, H70

\section{ABSTRACT}

The creation of companies has acquired great notoriety in the last ten years as a territorial instrument to counteract the dominant unemployment rates. Hence, this is one of the most decisive interventions between 1998-2018 by governments, which direct important efforts to promote and direct entrepreneurship. Business activity has a direct impact on a country's economic growth, so it requires intervention on uncertain factors to start a business that represent limits to productivity and business competitiveness. Consequently, the objective of this investigation was to determine the most problematic factors detected by the managers of the relevant companies in the Russian (centralized) and Spanish (decentralized) economies. To this end, a descriptive and comparative statistical analysis was carried out based on the annual survey of the Wold Economic Forum during the period 2011-2016. The main findings indicated that the most problematic factors for Spain in relation to Russia were five, among which the Inefficient Government Bureaucracy and the Tax Rates stand out. In contrast, the most problematic factors for Russia in relation to Spain were, above all, tax regulation and inflation.

Keywords: Economic development; international economy; economic policy; company; risk factor's.

JEL: F10, H70

\section{Introducción}

Las diferentes políticas económicas emprendidas por los gobiernos pueden favorecer o no el establecimiento de negocios en su territorio. A modo de ejemplo, podemos citar actuaciones que se centran en la fiscalidad empresarial bajo justificación específica, aquellas dirigidas a reforzar 
diferentes aspectos de la contratación, las que garantizan acceso a inputs y suministros o las que facilitan la liquidez necesaria para generar intercambios crecientes. Los resultados son el aumento de la renta, la riqueza empresarial y la generación del empleo.

Según Buendia Rice (2013, p.56):

Para que las empresas logren ventaja competitiva es necesario que se desenvuelvan en un ambiente propicio, ya sea contando con el capital humano necesario para satisfacer necesidades específicas, buena infraestructura económica, leyes que fomenten la competencia, incentivos para el comercio internacional o centros de investigación especializados que trabajen en conjunto con el sector privado. Esto pone un énfasis especial en el papel que debe desempeñar el Estado en el desarrollo de la competitividad nacional, ya que éste puede intervenir en la economía mediante políticas proactivas.

Si los países logran articular un entorno favorable a la creación de empresas innovadoras repercutirá directamente en el crecimiento económico (Giraldo, Bedoya y Vargas, 2009, p.101), y con ello, en el bienestar de sus habitantes. Por este motivo, esta investigación discurre sobre cómo determinar los factores más problemáticos detectados por los directivos y ejecutivos, de empresas relevantes en la economía de Rusia y España, para establecer negocios en su nación. Para ello, se realiza una comparativa de los factores más problemáticos para establecer un negocio, con el fin de evidenciar los factores más valorados por los directivos y ejecutivos en ambos países. Esta información será de gran utilidad para los gobiernos a la hora de elaborar políticas que atraigan y faciliten a las empresas a instalarse en su territorio. Además, se pretende dotar de evidencias a las futuras colaboraciones entre ambos países y, compartir las medidas que han realizado en aquellos factores que muestran una mayor o menor valoración.

\section{Metodología}

Al igual que otros trabajos académicos (Buendia, 2013; GarcíaOchoa, Bajo y Blázquez, 2012; Benzaquen, Carpio, Zegarra y Valdivia, 2010; Echebarría, 2006), se han tratado los datos de la encuesta anual realizada por Wold Economic Forum ${ }^{2}$. Esta institución es una fundación sin ánimo de lucro, con sede en Ginebra (Suiza), órgano independiente

2 Fuente: https://www.weforum.org/about/world-economic-forum (Consultado 15.04.2018). 
e imparcial, el cual proporciona una plataforma de contacto para que los líderes de diferentes colectivos (empresas, gobierno y sociedad civil) con el fin de poner en común iniciativas y caminar hacia el progreso económico.

Para completar las fuentes estadísticas existentes, Wold Economic Forum realiza una encuesta ${ }^{3}$ para medir conceptos críticos como: el apetito por el emprendimiento, el alcance de la brecha de habilidades y la incidencia de la corrupción.

Concretamente, en la edición de 2017 se captó las opiniones de 14.375 ejecutivos de empresas en más de 148 economías entre febrero y junio de 2017. Tras un proceso de depuración el total de respuestas se redujo a 12,775 respuestas de 133 economías.

La encuesta comprende 150 preguntas divididas en 15 secciones. La pregunta utilizada es del «Apartado II. Los factores más problemáticos para hacer negocios», la cual enumera una serie cerrada de 18 factores: acceso limitado a la financiación, inestabilidad del gobierno/golpes de Estado, regulaciones laborales restrictivas, crimen y robo, regulaciones en moneda extranjera, corrupción, infraestructura inadecuada, complejidad de las regulaciones fiscales, burocracia gubernamental ineficiente, altas tasas de impuestos, inadecuada formación de los trabajadores, inflación, pobre ética de trabajo en la fuerza de trabajo, mal sistema sanitario, inestabilidad política y capacidad insuficiente para innovar. Y enuncia «la selección de los cinco factores más problemáticos para hacer negocios en su país y clasificación de éstos del 1 (más problemático) a 5. Como segundo paso, para cada país, se suman los puntos por cada riesgo y se dividió por el total de puntos distribuidos entre todos los factores». En relación al horizonte temporal seleccionado, se optó por estudiar los últimos 6 años de información publicada (2011 a 2016), derivado por la importancia de conocer los principales elementos que dificultan establecer negocios en un país, así como, su estado actual y retrospectivo. Puntualizar que, se halló carencia de algunos factores desde 2010 hacía atrás.

3 Fuente: http://reports.weforum.org/global-competitiveness-index-2017-2018/appendix-c-the-executive-opinion-survey-the-voice-of-the-business-community/ (Consultado 17.04.2018). 


\section{Resultados}

En este apartado se procede, en primer lugar, a describir las principales características de Rusia y España, posteriormente se detallan los principales factores problemáticos para establecer un negocio en esos dos países.

\subsection{Descripción de los países}

Rusia es el país más extenso del mundo además, su población fue tres veces superior a la española. En cambio, el porcentaje de inmigrantes fue 4 puntos porcentuales superior en España. Esta realidad condiciona todas las políticas económicas que desee emprender su gobierno. Así mismo, Rusia presentó un impuesto sobre el valor añadido 3 puntos inferior y un impuesto sobre la renta bastante más bajo que España (Tabla 1).

Tabla 1. Principales características sociodemográficas de España y Rusia en 2017

\begin{tabular}{lll} 
& Rusia & España \\
Población & 143.990 .000 & 46.659 .302 \\
\hline \% de Hombres & $46,64 \%$ & $49,04 \%$ \\
\hline \% de Mujeres & $53,71 \%$ & $50,96 \%$ \\
\hline \% de inmigrantes & $8,09 \%$ & $12,75 \%$ \\
\hline Extensión & 17.098 .250 & 505.940 \\
\hline Moneda & Rublos rusos & Euro \\
\cline { 2 - 3 } & 1 EURO = 78,5871 RUBLOS \\
\hline Impuesto sobre el valor añadido (tipo máximo) & $18 \%$ & $21 \%$ \\
\hline Impuesto sobre la renta de los trabajadores (tipo máximo) & $13 \%$ & $43,5 \%$ \\
\hline
\end{tabular}

Fuente: Datosmacro ${ }^{4}$

En el gráfico 1 se observa que España presentó un PIB per cápita más del doble que Rusia, además, la evolución rusa ha sido decreciente mientras que España se ha mantenido estable. En cambio, la tasa de desempleo (gráfico 2) española fue cuatro veces mayor que la rusa, presentando una evolución ascendente hasta el 2015, mientras que la rusa se mantuvo en niveles muy similares durante el periodo de analizado. Lo que pone de relieve que no se manifiesta una relación directa positiva entre PIB per cápita y tasa de

4 Fuente: https://datosmacro.expansion.com (Consultado 24/08/2018). 
paro. Ciertamente, estos elementos deberían influir en la percepción de los empresarios a la hora de establecer un negocio en estos territorios.

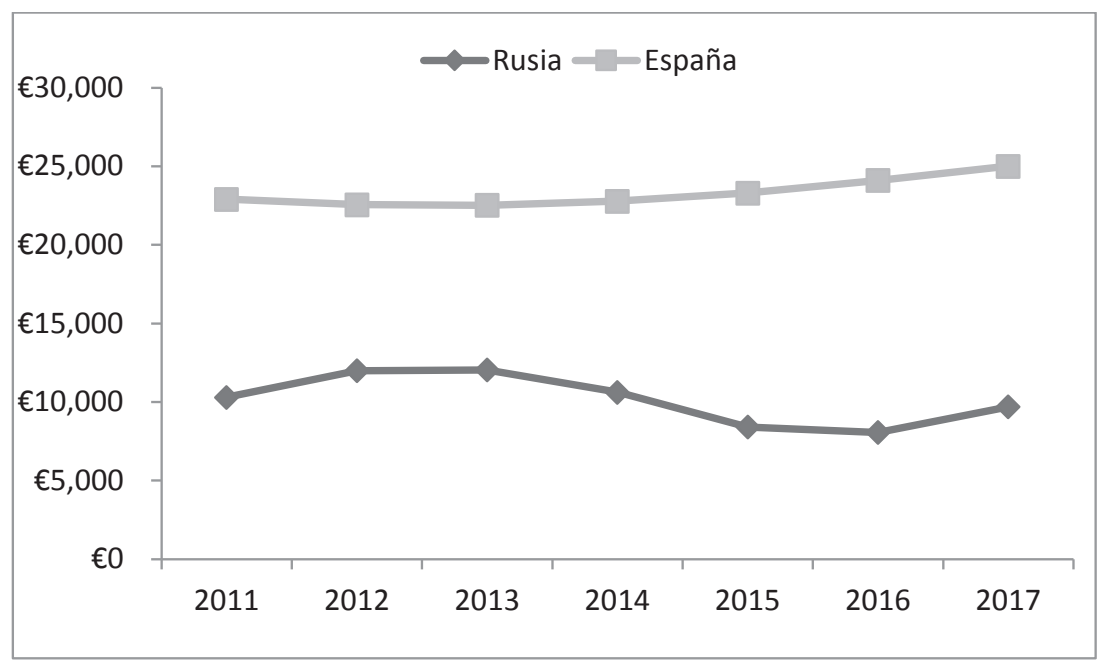

Gráfico 1. Evolución del PIB Per Cápita de España y Rusia Fuente: Datosmacro ${ }^{5}$

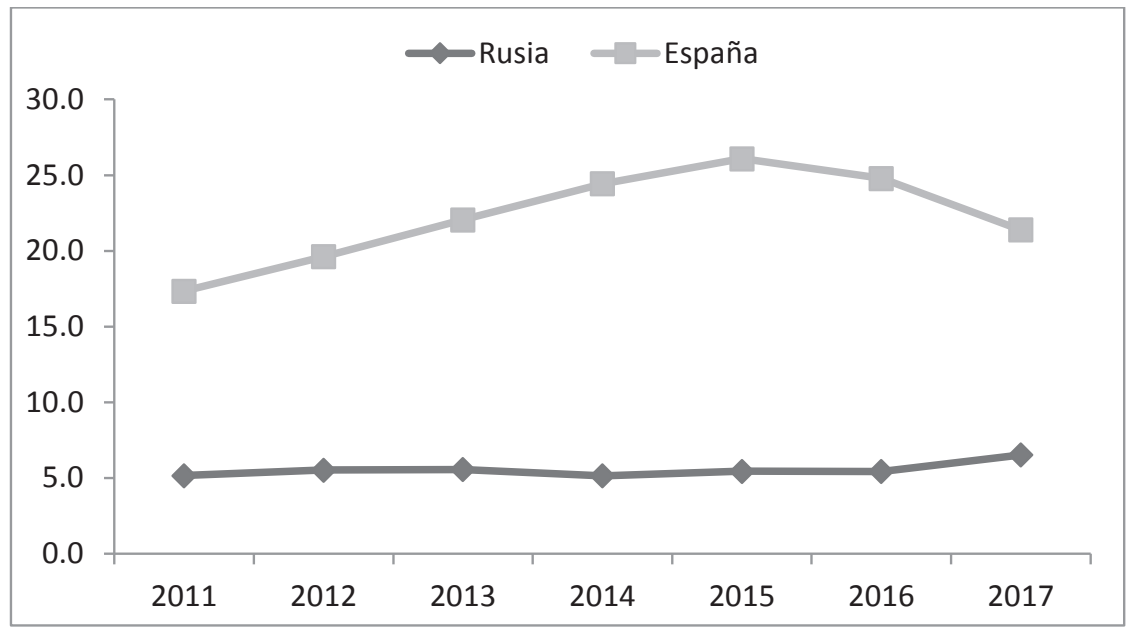

Gráfico 2. Evolución la tasa de paro de España y Rusia Fuente: Banco Mundial ${ }^{6}$

5 Fuente: https://datosmacro.expansion.com (Consultado 24/08/2018).

6 Fuente: https://datos.bancomundial.org/indicador/SL.UEM.TOTL.ZS?end=2017\&start=2017\&view=map (Consultado $24 / 08 / 2018)$. 


\subsection{Factores más problemáticos para establecer negocios}

En un intento de delimitar los principales elementos o causas que suponen una dificultad para hacer negocios en España y Rusia a partir de los informes The Global Competitiveness Report ${ }^{7}$ de los años 2012-2013 a 2017-2018 y tras la explicación metodológica sobre el Wold Economic Forum, añadir que los resultados que se ofrecen sobrepasan el valor 5, al trabajar con una media del total de elementos valorados. A continuación, se exponen los diferentes factores problemáticos por orden de mayor a menor valoración manifestada por los directivos y ejecutivos de ambos países:

Como se observa en la Tabla 2, en 2016 el factor más problemático para hacer negocios en España fue «Ineficiente burocracia gubernamental», además se ha mantenido entre los tres principales durante los seis años de estudio.

Tabla 2. Valoración media de los factores más problemáticos en España

\begin{tabular}{lrrrrrr}
\multicolumn{1}{c}{ Factores problemáticos } & $\mathbf{2 0 1 1}$ & $\mathbf{2 0 1 2}$ & $\mathbf{2 0 1 3}$ & $\mathbf{2 0 1 4}$ & $\mathbf{2 0 1 5}$ & $\mathbf{2 0 1 6}$ \\
Burocracia gubernamental ineficiente & 13,5 & 17,6 & 13,6 & 15,9 & 14,0 & 15,2 \\
Tasas de impuestos & 9,4 & 10,5 & 12,8 & 17,3 & 14,5 & 15,1 \\
Regulaciones laborales restrictivas & 16,1 & 14,0 & 14,4 & 14,7 & 13,2 & 13,5 \\
Capacidad insuficiente de innovar & 11,9 & 10,1 & 8,0 & 10,0 & 12,1 & 11,3 \\
Acceso a financiamiento & 27,8 & 27,7 & 26,7 & 18,9 & 11,0 & 9,6 \\
Inestabilidad de la política & 2,3 & 1,6 & 1,5 & 3,5 & 9,1 & 7,4 \\
Inadecuada formación de los trabajadores & 3,4 & 2,5 & 2,5 & 4,0 & 6,1 & 6,9 \\
Complejidad de las regulaciones fiscales & 5,0 & 4,9 & 6,7 & 4,4 & 4,4 & 5,4 \\
Corrupción & 3,7 & 6,2 & 9,0 & 7,4 & 7,4 & 5,0 \\
Inestabilidad del gobierno / golpes de estado & 0,0 & 0,2 & 0,7 & 0,0 & 1,4 & 3,4 \\
Pobre ética de trabajo en la mano de obra & 3,0 & 2,3 & 2,9 & 1,9 & 3,7 & 3,2 \\
Suministro inadecuado de infraestructura & 1,4 & 1,0 & 0,4 & 1,3 & 2,5 & 2,0 \\
Inflación & 2,0 & 0,8 & 0,3 & 0,0 & 0,2 & 1,0 \\
Mal sistema sanitario & 0,5 & 0,1 & 0,4 & 0,6 & 0,0 & 0,9 \\
Regulaciones en moneda extranjera & 0,0 & 0,0 & 0,2 & 0,3 & 0,4 & 0,1 \\
Crimen y robo & 0,0 & 0,5 & 0,0 & 0,0 & 0,0 & 0,0 \\
\hline
\end{tabular}

Fuente: The Global Competitiveness Report 2016/17, 2015/16, 2014/15, 2013/14, 2012/11.

7 Apartado que ofrece los datos sobre los factores más problemáticos para hacer negocio. 
En cambio, para Rusia este elemento ocupó el segundo puesto en 2011 y se redujo hasta alcanzar el séptimo lugar en 2016 (tabla 3). En el Gráfico 3 se comprueba que Rusia presentó un resultado inferior a España en todos los años objeto de estudio y mientras que en España presenta una evolución en forma de diente de sierra, que marca un punto final superior al inicial, lo que marca a este como el más problemático en los dos últimos años, si bien presentó un pico en 2012 con 17.6, que no ha vuelto a superarse. Sin embargo, en el caso de Rusia hay una pendiente negativa que indica una mejor valoración del ítem, que se ha reducido de forma acusada hasta llegar a un mínimo 6 en 2015.

Tabla 3. Valoración media de los factores más problemáticos en Rusia

\begin{tabular}{lrrrrrr}
\multicolumn{1}{c}{ Factores problemáticos } & $\mathbf{2 0 1 1}$ & $\mathbf{2 0 1 2}$ & $\mathbf{2 0 1 3}$ & $\mathbf{2 0 1 4}$ & $\mathbf{2 0 1 5}$ & $\mathbf{2 0 1 6}$ \\
Tasas de impuestos & 9,3 & 13,0 & 12,3 & 12,3 & 13,3 & 13,1 \\
Corrupción & 20,5 & 19,1 & 14,3 & 14,3 & 10,9 & 13,1 \\
Acceso a financiamiento & 10,0 & 9,2 & 11,7 & 11,7 & 10,7 & 10,2 \\
Inflación & 4,1 & 7,1 & 7,6 & 7,6 & 13,8 & 10,1 \\
Regulaciones fiscales & 6,0 & 10,7 & 10,8 & 10,8 & 7,7 & 9,3 \\
Inadecuada formación de los trabajadores & 7,1 & 6,6 & 7,3 & 7,3 & 4,6 & 7,4 \\
Burocracia gubernamental ineficiente & 11,9 & 9,8 & 8,2 & 8,2 & 6,0 & 6,1 \\
Inestabilidad de la política & 4,5 & 1,8 & 2,8 & 2,8 & 6,7 & 5,5 \\
Pobre ética de trabajo en la fuerza de trabajo & 3,1 & 4,2 & 4,8 & 4,8 & 3,6 & 5,2 \\
Suministro inadecuado de infraestructura & 4,3 & 4,5 & 4,6 & 4,6 & 4,3 & 3,9 \\
Inestabilidad del gobierno / golpes de estado & 1,6 & 1,6 & 2,0 & 2,0 & 3,1 & 3,8 \\
Capacidad insuficiente para innovar & 6,5 & 4,3 & 4,0 & 4,0 & 3,6 & 3,3 \\
Regulaciones en moneda extranjera & 1,1 & 0,8 & 2,2 & 2,2 & 5,2 & 3,2 \\
Regulaciones laborales restrictivas & 2,3 & 2,3 & 3,6 & 3,6 & 2,7 & 2,4 \\
Crimen y robo & 5,9 & 4,3 & 2,8 & 2,8 & 2,9 & 2,1 \\
Mal sistema sanitario & 1,7 & 0,9 & 1,0 & 1,0 & 1,0 & 1,4 \\
\hline
\end{tabular}

Fuente: The Global Competitiveness Report 2016-2017, 2015-2016, 2014-2015. 


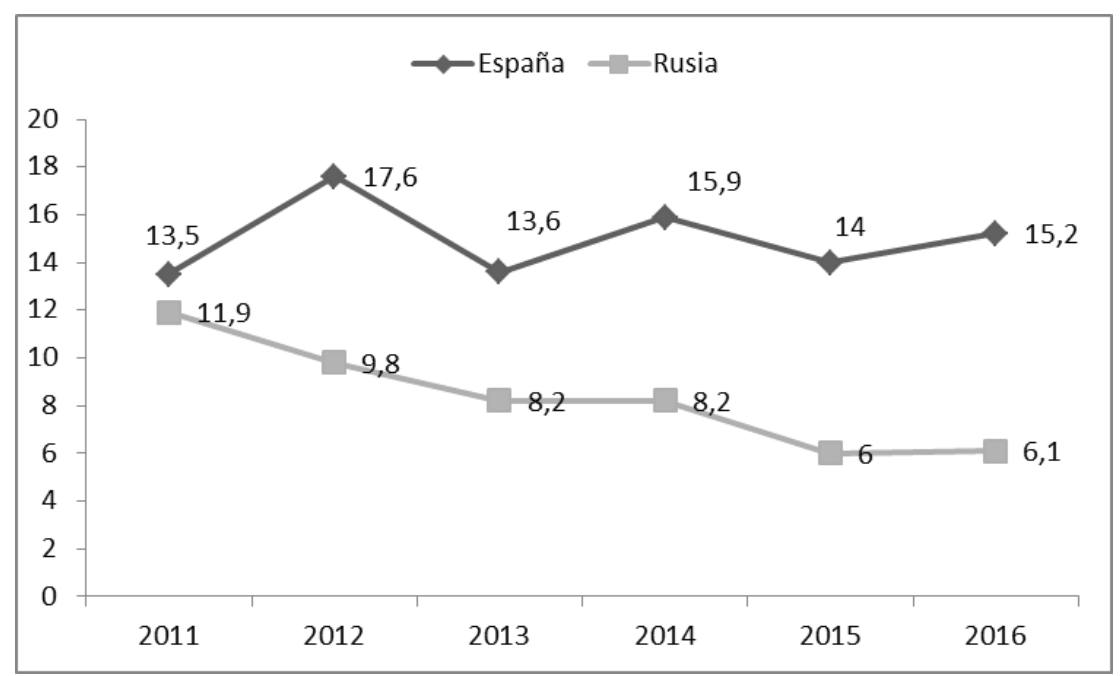

Gráfico 3. Evolución de la valoración sobre la Ineficiente Burocracia Gubernamental Fuente: The Global Competitiveness Report 2016/17, 2015/16, 2014/15, 2013/14, 2012/11

El siguiente elemento que mostró una valoración más elevada en 2016 para España fue «Tasas de Impuestos», pues ha ido incrementándose hasta 2014, donde igualmente ocupó el segundo lugar. Aunque con una valoración ligeramente inferior (Gráfico 4), también significó para Rusia el segundo factor con peor resultado entre 2012 y 2016. Aunque el entramado de tipos de impuesto en muy amplio para ambos países, resaltamos que tanto el impuesto sobre el valor añadido y el de la renta del trabajo, los cuales se expusieron en la tabla 1 , son bastante más inferiores en Rusia.

Las «regulaciones laborales restrictivas» suponen la tercera variable más problemática para España en 2016 y se ha mantenido entre los tres principales durante el horizonte temporal analizado (Tabla 2). En cambio, para Rusia este factor no presentó una valoración especialmente elevada, lo que llevó a situarse entre los cuatro últimos puestos entre 2011 y 2016 (Tabla 3). Por otro lado, es preciso resaltar las amplias diferencias halladas en la valoración de este factor entre ambos países, lo que manifestó que los empresarios y directivos españoles perciben que la regulación laboral restrictiva fue un factor de riesgo elevado (Gráfico 5) y en mayor medida en que lo hicieron los rusos. 


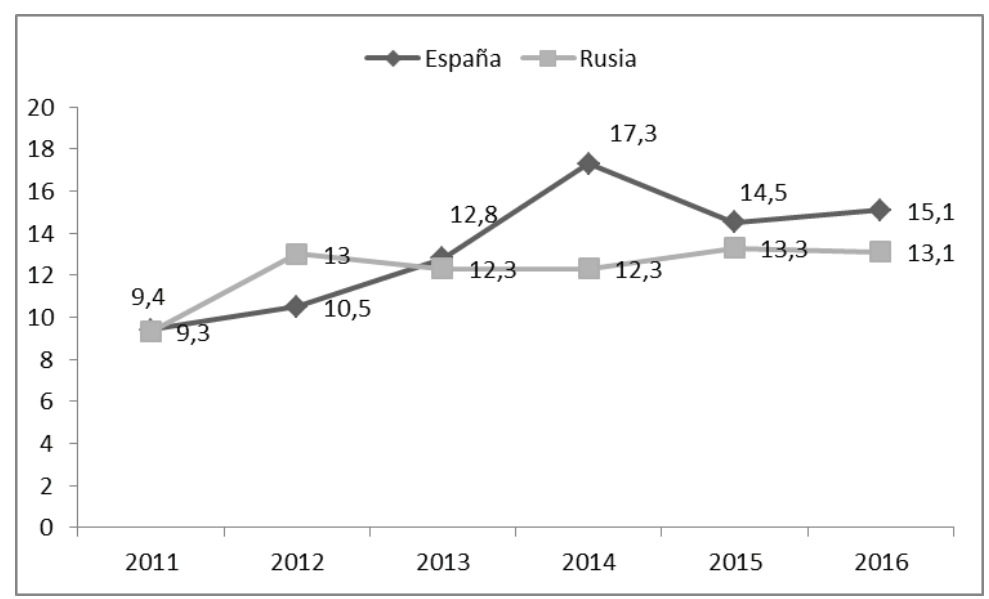

Gráfico 4. Evolución de la valoración sobre las Tasas de Impuestos

Fuente: The Global Competitiveness Report 2016/17, 2015/16, 2014/15, 2013/14, 2012/11

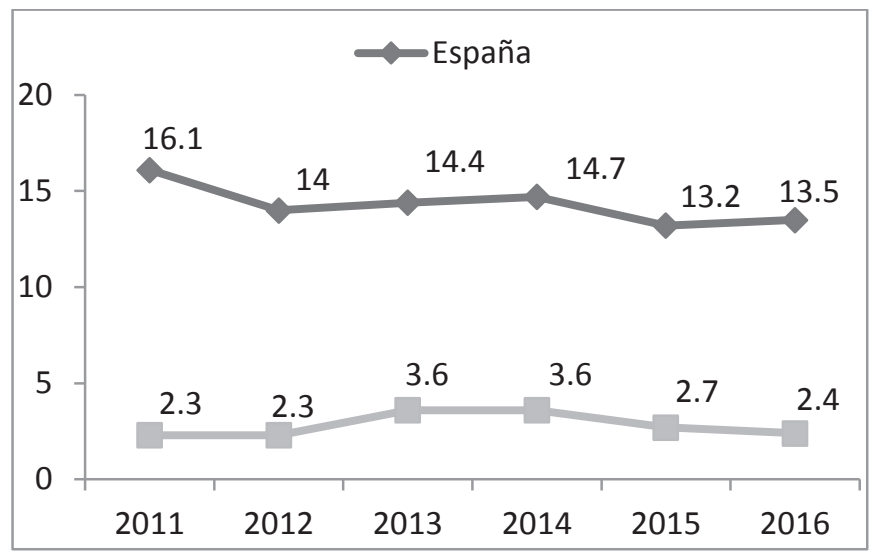

Gráfico 5. Evolución de la valoración sobre Regulaciones Laborales Restrictivas Fuente: The Global Competitiveness Report 2016/17, 2015/16, 2014/15, 2013/14, 2012/11

Los empresarios y directivos españoles valoraron como el cuarto factor problemático la «Capacidad insuficiente de innovar» en 2011, 2015 y 2016 (Tabla 2). A pesar de haber sido la quinta variable más valorada por los rusos en 2011, fue reduciéndose considerablemente (Gráfico 6). Los resultados evidencian que es un factor problemático mayor en España que en Rusia. 
Pensamiento Crítico Vol. 23. $\mathrm{N}^{\circ} 2$

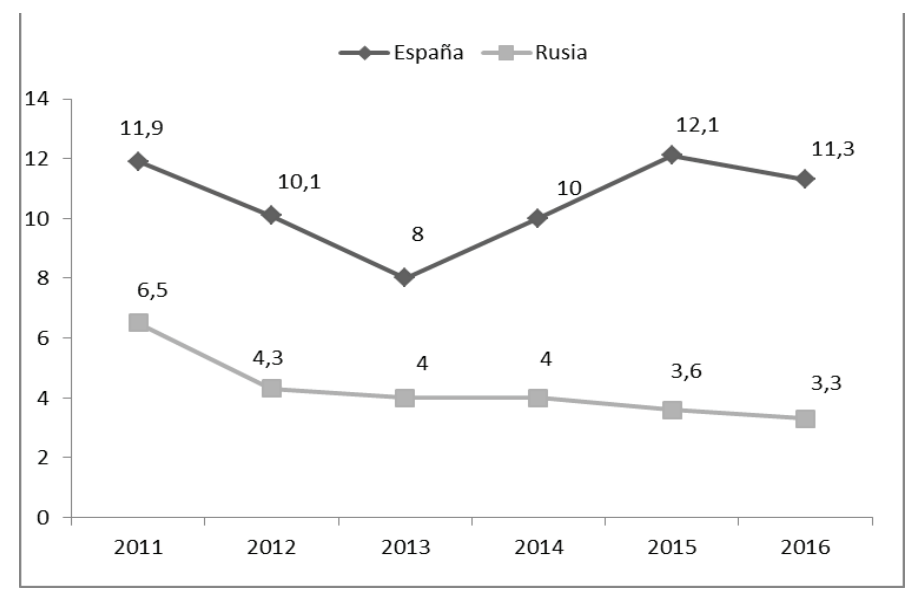

Gráfico 6. Evolución de la valoración sobre la Capacidad insuficiente de Innovar Fuente: The Global Competitiveness Report 2016-17, 2015-16, 2014-15, 2013-14, 2012-11

Hasta el 2014 el «Acceso a Financiación» fue el principal factor de riesgo en España, entendible con la recesión económica y financiera iniciada en el segundo semestre de $2008^{8}$ cuyo impacto en el acceso a la financiación aún no han quedado completamente resueltas, aunque sí han disminuido. A partir de ese año se iniciaron una serie de actuaciones por parte del Banco Central Europeo para garantizar la estabilidad de precios a medio plazo y gestionar la liquidez con el fin de mejorar la solvencia del sistema financiero (Buitrago-Esquinas y Romero-Landa, 2013, p.281). Además, en 2012 España recibió ayuda del Mecanismos Europeo de Estabilidad $^{9}$ para recapitalizar su sector bancario. Tal como evidencia el Gráfico 7 este contexto de crisis financiera se tradujo en una valoración elevada de los directivos y ejecutivos españoles que se ha ido reduciendo, considerablemente, en los dos últimos años, hasta alcanzar el quinto puesto de los factores más problemáticos (coincidiendo con valores muy similares a los rusos). Ciertamente, la crisis alcanzó una escala mundial, pero esta no ha impactado en la variable analizada de la misma forma en España y Rusia. Aunque los resultados entre ambos países son muy

8 Fuente: https://europa.eu/european-union/topics/economic-monetary-affairs_es (Consultado 20.04.2018).

9 Fuente: http://www.consilium.europa.eu/es/policies/financial-assistance-eurozone-members/ (Consultado 19.04.2018). 
dispares hasta 2015, es necesario recalcar que fue el tercer elemento con mayor valoración para Rusia en 2011, 2013, 2014 y 2016.

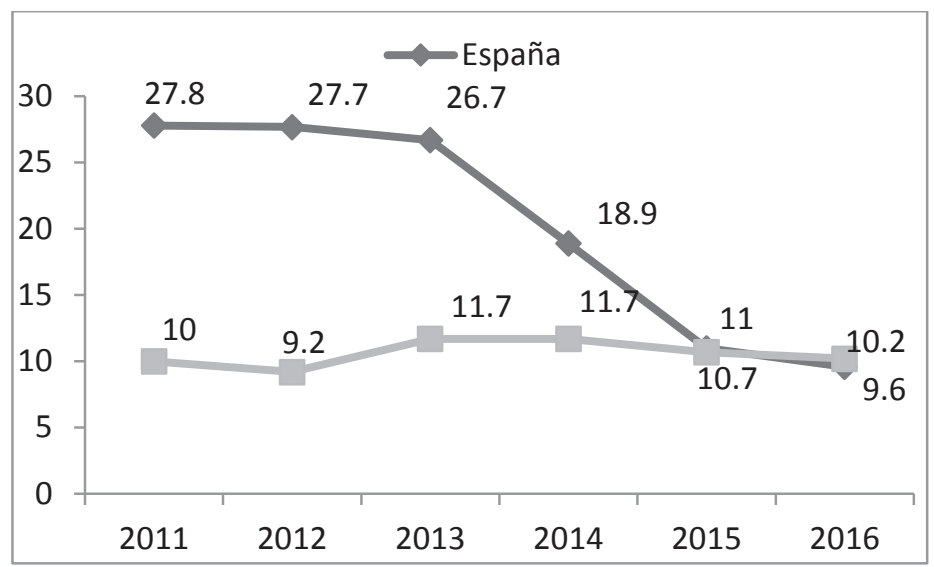

Gráfico 7. Evolución de la valoración sobre el Acceso Financiación

Fuente: The Global Competitiveness Report 2016-17, 2015-16, 2014-15, 2013-14, 2012-11

Para España, la «Inestabilidad Política» fue un elemento que hasta el 2013 ocupó el décimo lugar (Tabla 2), pero en los dos últimos años de estudio ascendió hasta el sexto coincidiendo con las elecciones al Parlamento Europeo donde se presentó un nuevo partido político, «Podemos», que ha introducido elementos que para una parte importante del tejido empresarial es signo de inestabilidad. Posteriormente, un segundo partido comenzó su auge «Ciudadanos». Esta situación junto con las denuncias de corrupción de los partidos tradicionales (Partido Popular y Partido Socialista Obrero Español) y el debate introducido sobre la regeneración política, provocaron que en las últimas elecciones regionales estos nuevos partidos tuvieran un papel decisivo en la formación de gobiernos regionales en las 17 comunidades autónomas (Gómez-Fortes y Urquizu, 2015) y, a continuación, en las elecciones generales (Junio de 2016).

A pesar que desde 2014 España muestra unos datos superiores a Rusia (Gráfico 8), la evolución de esta variable ha sido muy similar para ambos, coinciden en que en dicho año aumento su valoración como factor problemático. El gobierno ruso también ha estado salpicado por casos de 
corrupción ${ }^{10}$, además de que el mensaje nacionalista tradicional de sus dirigentes ha perdido fuerza en la población más joven (Dir. Garcés, 2017). En el último año la mejora producida es más intensa en España que en Rusia, si bien aquella marca una distancia elevada por lo que las circunstancias existentes en cada caso generan mayor desconfianza en el tejido empresarial español que en el ruso.

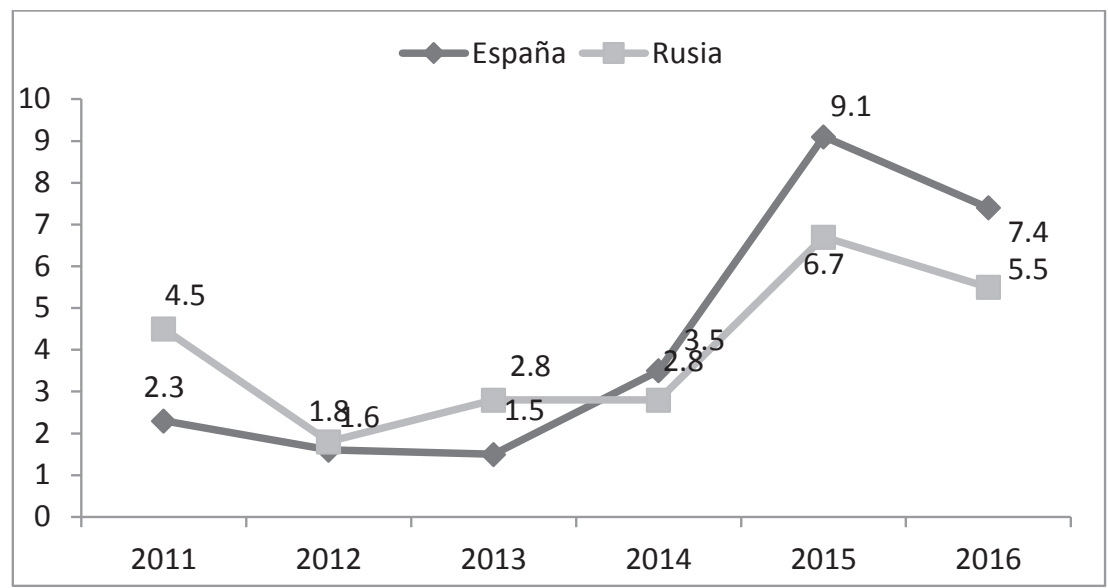

Gráfico 8. Evolución de la valoración la Inestabilidad Política

Fuente: The Global Competitiveness Report 2016-17, 2015-16, 2014-15, 2013-14, 2012-11

La importancia de la «adecuación de la Formación» de la mano de obra a las necesidades de las empresas ocupó, tanto para España como para Rusia, los puestos intermedios de las Tablas 2 y 3 en el horizonte temporal analizado. Aunque se ha ido incrementando con el transcurso de los años, los directivos y ejecutivos españoles mostraron una valoración ligeramente inferior a los rusos (Gráfico 9). El Gobierno español, intenta dar respuesta a este problema, entre las acciones realizadas destaca el Informe sobre prospección y detección de necesidades formativas realizado por el Observatorio de las Ocupaciones del Servicio Público de Empleo Estatal $^{11}$, así como la introducción de estrategias de impulso al empleo y las prácticas laborales en todas la universidades españolas.

10 El hartazgo con la corrupción insta a protestas jóvenes, aumenta la actitud más reivindicativa de la ciudadanía (Fuente: https://elpais.com/internacional/2017/03/27/actualidad/1490638159_254860. html).

11 Fuente: https://www.sepe.es/contenidos/que_es_el_sepe/observatorio/que_es_observatorio.html (Consultado 


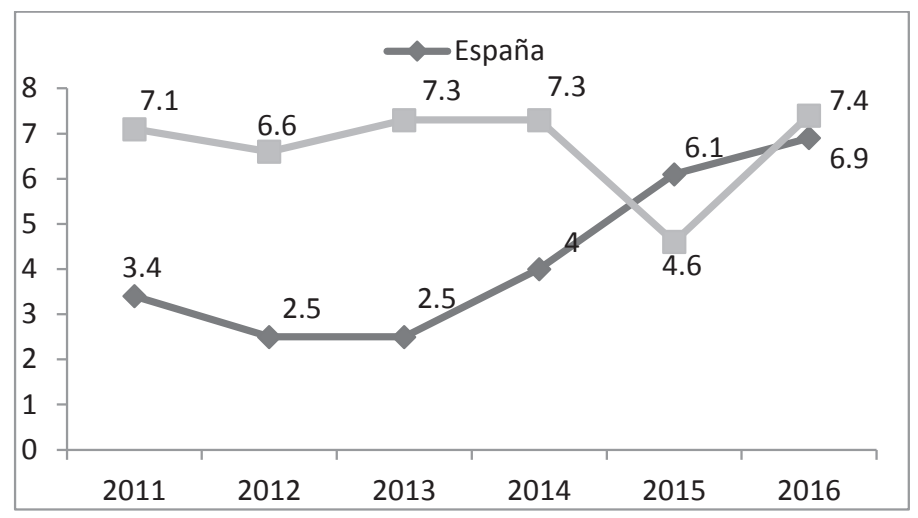

Gráfico 9. Evolución de la valoración la Inadecuada Formación de los Trabajadores Fuente: The Global Competitiveness Report 2016-17, 2015-16, 2014-15, 2013-14, 2012-11

En el caso de España, la «Regulación Fiscal» también abarcó los puestos centrales de la Tabla 2 en el periodo estudiado. Aunque ambos países partieran de resultados adyacentes, se evidencia una valoración superior para los directivos y ejecutivos rusos (Gráfico 10). Según Musaeva, Zaidullaevna y Bashirovna (2015), existen importantes diferencias regionales en Rusia derivada de la descentralización del sistema presupuestario.

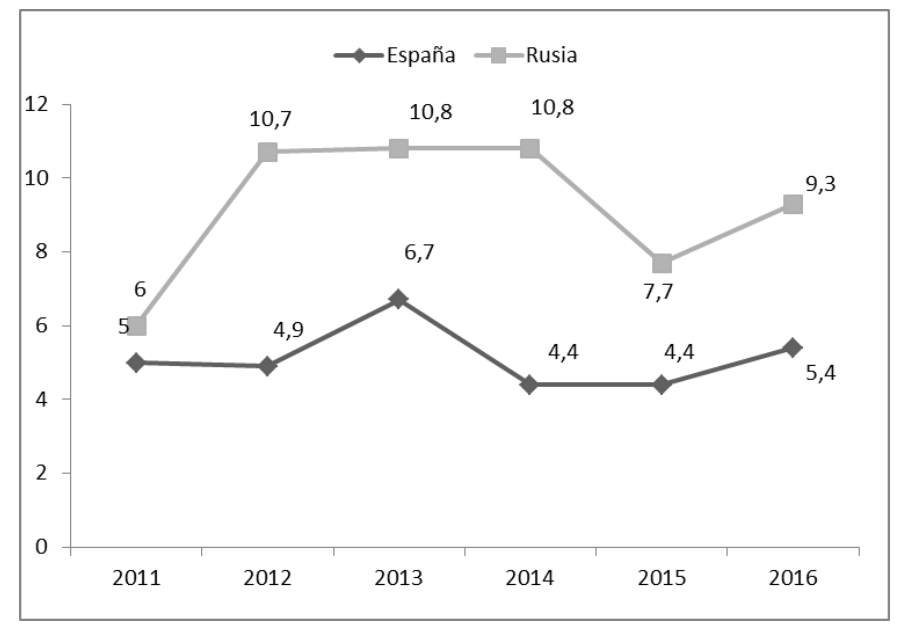

Gráfico 10. Evolución de la valoración de la Complejidad de la Regulación Fiscal Fuente: The Global Competitiveness Report 2016-17, 2015-16, 2014-15, 2013-14, 2012-11

19.04.2018). 
En la actualidad la valoración de la «Corrupción» como factor problemático para establecer un negocio es España ocupa el noveno lugar y aunque entre 2013 y 2014 obtuvo una media un poco más elevada no llegó a entrar dentro de los 5 elementos más problemáticos (Tabla 2). En cambio, para Rusia es la variable con mayor resultado durante todos los años estudiados (menos el 2015), a pesar de que se haya ido reduciendo (Gráfico 11). Según la información facilitada por Datosmacro ${ }^{12}$, que elabora un índice de Percepción de la Corrupción entre 180 países (ranking de menor a mayor), en 2017 España ocupa el puesto 42 y Rusia el 135. Autores, como Auerbach y Azariadis (2015) o Hickey (2012), consideran clave para lograr un desarrollo económico y social exitoso la necesidad de eliminar la corrupción, combatir la economía sumergida y minimizar el impacto negativo de los gobiernos en los negocios.

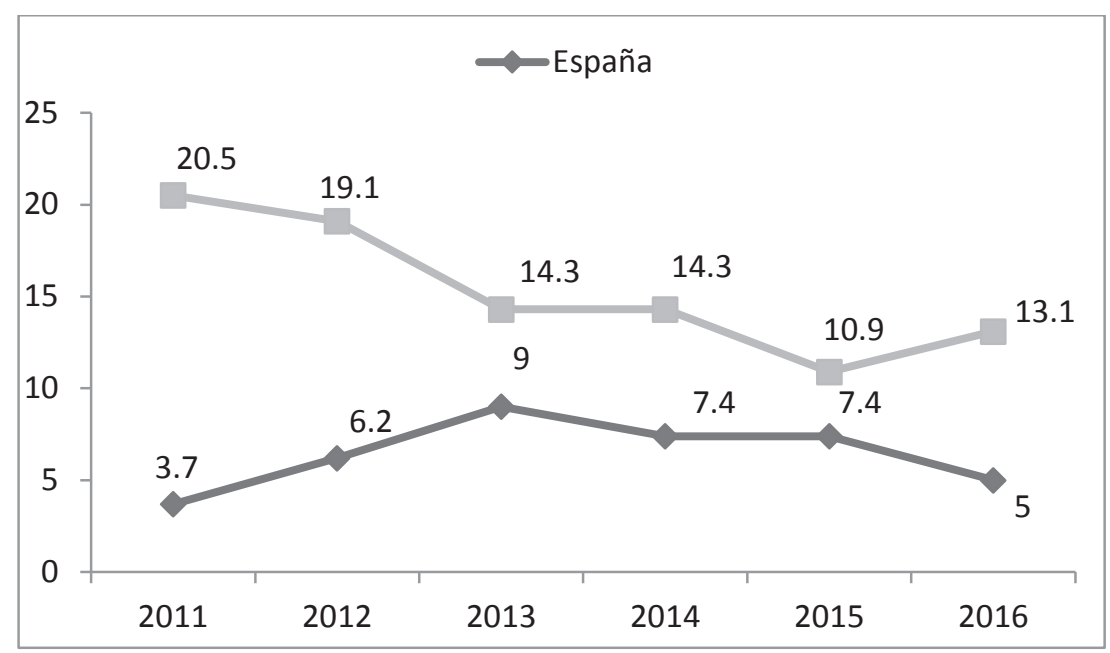

Gráfico 11. Evolución de la valoración de la Corrupción

Fuente: The Global Competitiveness Report 2016-17, 2015-16, 2014-15, 2013-14, 2012-11

Hasta aquí se han mostrado los factores problemáticos que exhibieron una valoración superior o igual a 5 para para ambos países en 2016. A continuación, se procede a comentar la evolución del factor «Inflación», 
pues debe ser tratado de manera individual por la importancia que adquiere para Rusia (Gráfico 12).

España forma parte de la zona euro de la Unión Europea, por lo que tiene cedida su competencia de política monetaria al Banco Central Europeo. Dicha política tiene como objetivo "mantener a medio plazo las tasas de inflación por debajo del 2\%, pero próximas a este valor"13. El Banco Central de la Federación Rusia ${ }^{14}$ es el órgano encargado de gestionar la política monetaria de Rusia, dentro sus objetivos está el de alcanzar una inflación entorno al 4\% para 2017.

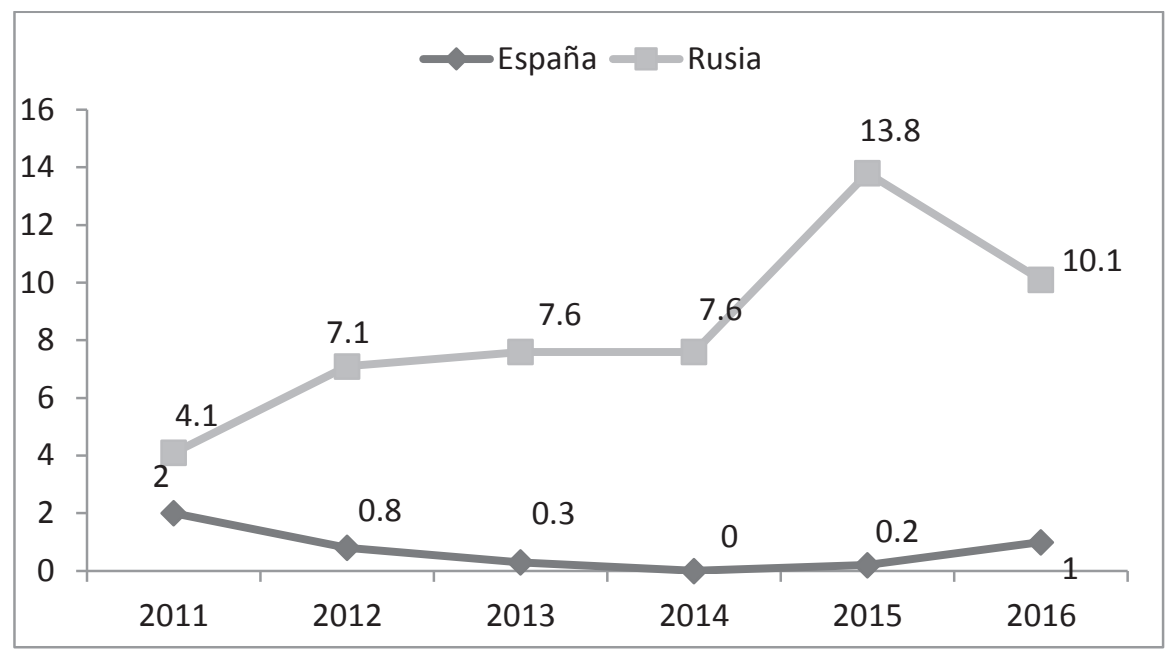

Gráfico 12. Evolución de la valoración de la Inflación

Fuente: The Global Competitiveness Report 2016-17, 2015-16, 2014-15, 2013-14, 2012-11

Como se observa en el Gráfico 12, la tasa de inflación de España se ha ido reduciendo mientras que la rusa incrementó, además de evidenciar un diferencial muy amplio entre ambos países. Esta situación ha debido de influir en la valoración de los directivos y ejecutivos sobre la inflación como factor problemático para establecer un negocio, pues se observa analogía entre la senda seguida por la inflación y la valoración de la mis-

13 Fuente: https://www.bde.es/bde/es/areas/polimone/estrategia/La_estrategia_del_BCE.html (Consultado 27.05.2018)

14 Fuente: https://www.cbr.ru/eng/today/ (Consultado 19.04.2018). 
ma. Durante el periodo de crisis la inflación ha caído por lo que el Banco Central Europeo ha llevado a cabo medidas para incentivar el consumo.

Aunque ello puede representar un error de concepto, que es extraño entender en la valoración de directivos y empresarios. Para estos la subida de precios es un factor que alimenta la oferta y la bajada la contiene. Es más, el nivel de precios de España presenta una desinflación que representa una respuesta de la oferta ante la dificultad de captar demanda, reflejando más bien un intento de representar un cambio de paradigma donde la necesidad de bajar precios obliga a la oferta a un ajuste continuo de sus mecanismos de producción o prestación de servicios para adaptarse a ello, lo cual genera un entorno empresarial probablemente más inestable.

Así y todo, aunque la valoración problemática en España responda a un nivel excesivamente bajo, el nivel inflacionista presentado por Rusia frente al objetivo planteado no injusticia su valoración, sino todo lo contrario. Si es importante señalar como es negativo el hecho de no cumplir el objetivo, tanto por encima como por debajo, y ambos tienen riesgos importantes para el tejido empresarial.

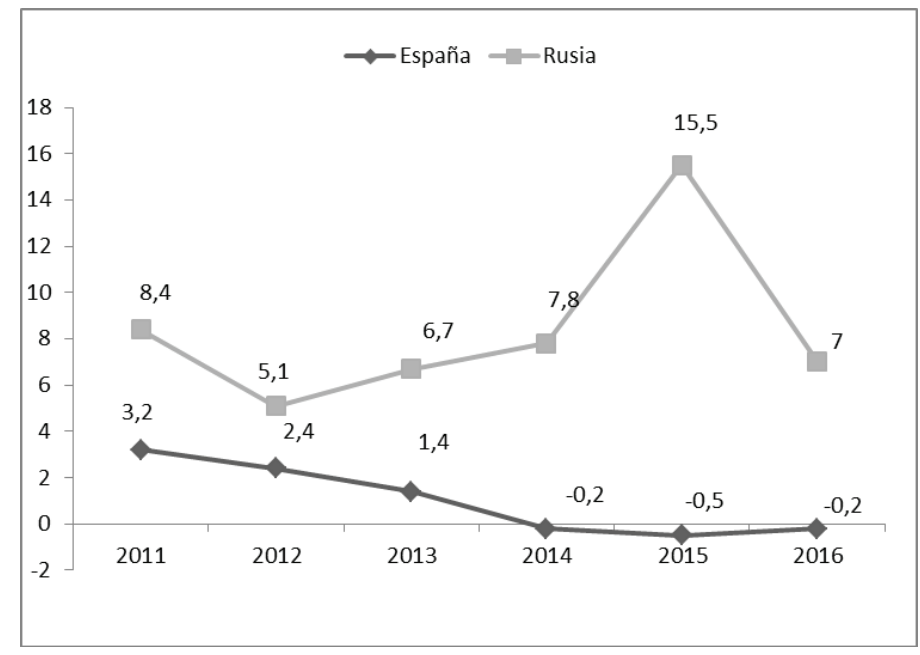

Gráfico 13. Evolución de la Inflación en España y Rusia Fuente: Banco Mundial. ${ }^{15}$

15 Fuente: https://datos.bancomundial.org/indicador/fp.cpi.totl.zg?end=2016\&start=2015 (Consultado 19/04/2018). 
Por último, los factores problemáticos menos valorados en ambos países fueron: inestabilidad del gobierno / golpes de estado, pobre ética de trabajo en la mano de obra, suministro inadecuado de infraestructura, mal sistema sanitario, regulaciones en moneda extranjera, crimen y robo (Tablas 2 y 3 ).

\section{Conclusiones}

Los cinco factores problemáticos más valorados por las empresas españolas son (por orden de mayor a menor) en 2016: burocracia gubernamental ineficiente, tasas de impuestos, regulaciones laborales restrictivas, ineficiente capacidad de innovar y acceso a la financiación (Tabla 4). El primero y segundo han ido incrementando en el horizonte temporal analizado, mientras que los otros se redujeron ligeramente.

Tabla 4. Valoración media de los 5 factores más problemáticos para negocios en España

Los 5 factores problemáticos más valorados en 2016

España

Diferencia con Rusia

Burocracia gubernamental ineficiente

15,2

9,1

Tasas de impuestos

15,1

2,0

Regulaciones laborales restrictivas

13,5

11,1

Capacidad insuficiente de innovar

11,3

8,0

Acceso a financiamiento

9,6

$-0,6$

Fuentes: The Global Competitiveness Report 2017-2018

Los directivos y ejecutivos de empresas rusas identificaron como los 5 principales factores problemáticos para establecer un negocio en 2016 (por orden de mayor a menor): corrupción, tasas de impuestos, acceso a financiación, inflación y regulaciones fiscales (Tabla 5). El primero y el tercero se han mantenido estables, ciertamente la corrupción y el acceso a financiación descienden, pero la inflación aumenta.

Entre las principales diferencias entre ambos países, destacar cinco:

- Ambos países comenzaron en 2011 con una valoración elevada de la «Ineficiente burocracia gubernamental» pero Rusia ha logrado reducirla mientras que España no. 
- Para los directivos y ejecutivos rusos la «Regulación laboral restrictiva» no supone un factor problemático de especial relevancia y sí, la «Regulación Fiscal» y la «Corrupción».

- La «Inflación» no parece constituir un elemento problemático para España, a pesar de los peligros empresariales y para el mercado de la situación; mientras que la valoración de los ejecutivos rusos se ha ido incremento.

- Los resultados evidenciaron que la «Capacidad insuficiente de innovar» constituyó un factor problemático mayor en España que en Rusia. Además, los directivos rusos redujeron su valoración con el paso de los años mientras que los españoles la aumentaron.

- La valoración es similar ante la «Inestabilidad Política e Inadecuada Formación de los Trabajadores».

Tabla 5. Valoración media de los 5 factores más problemáticos para negocios en Rusia

\begin{tabular}{lcc} 
Los $\mathbf{5}$ factores problemáticos más valorados en $\mathbf{2 0 1 6}$ & Rusia & Diferencia con España \\
Corrupción & 13,1 & 8,1 \\
Tasas de impuestos & 13,1 & $-2,0$ \\
Acceso a financiamiento & 10,2 & 0,6 \\
Inflación & 10,1 & 9,1 \\
Regulaciones fiscales & 9,3 & 3,9 \\
\hline
\end{tabular}

Fuentes: The Global Competitiveness Report 2017-2018

En definitiva, para ambos países existen tantos factores problemáticos para establecer negocios que se mantienen regulares como irregulares en el tiempo. De manera que existen diferencias y similitudes, profundizar en sus causas permitirá obtener resultados de gran utilidad para mejorar la aplicación de las políticas económicas que fomenten el emprendimiento de España y Rusia, y con ello, generar sinergias para trabajar conjuntamente. 


\section{Referencias Bibliográficas}

Auerbach, Jan U. y Azariadis, Costas. (2015). Property Rights, Governance, and Economic Development. En Review of Development Economics, Vol.19, No.2, abril-mayo 2015, p. 210-20. DOI: https://doi.org/10.1111/rode.12138

Benzaquen, Jorge; Carpio, Luis Alfonso; Zegarra, Luis Alberto y Valdivia, Christian Alberto (2010). Un Índice regional de competitividad para un país. En Revista Cepal, Vol. 102, diciembre 2010, p.69-86. Disponible en https://repositorio.cepal.org/handle/11362/11417

Buendia Rice, Edgar Alejandro (2013). El papel de la Ventaja Competitiva en el desarrollo económico de los países. En Análisis Económico, Vol.28, No.69, septiembre-diciembre 2013, p.56-78. Disponible en http://www.analisiseconomico.azc.uam.mx/index.php/rae/article/view/144

Buitrago Esquinas, Eva María y Romero Landa, Luis Benjamín (2013). Economía de la Unión Europea, Ed. Pirámide, Madrid.

Dir. Garcés G. (2017). Centro de Economía Internacional de Libertad y Desarrollo Rusia: Estabilidad Política Amenazada y Débil Recuperación Económica. En Economía Internacional al Instante, Vol. 910.

Echebarría, Koldo (2006). Caracterización empírica de las burocracias latinoamericanas: configuraciones y roles en el proceso de elaboración de políticas públicas. En Revista del CLAD Reforma y Democracia, Vol. 34, p.1-11. Disponible en http://www.redalyc.org/html/3575/357533666004/

García-Ochoa Mayor, Mónica; Bajo Davó, Nuria y Blázquez de la Hera, María Luisa (2012). La innovación tecnológica como variable determinante en la competitividad de los países. En Revista de Economía Mundial, Vol.31, p.137166. Disponible en https://search.proquest.com/docview/1293627004?pqorigsite $=$ gscholar

Giraldo Múnera, Adriana; Bedoya Bermúdez, Giovanni y Vargas Restrepo, Carlos Mario (2009). Principales limitaciones del empresarismo que afectan el desarrollo económico y social del país. En Revista Escuela de Administración de Negocios, Vol. 66, p. 99-112. DOI: https://doi.org/10.21158/01208160. n66.2009.476

Gómez Fortes, Braulio y Urquizu, Ignacio (2015). Political Corruption and the End of two-party system after the May 2015 Spanish Regional Elections. En Regional \& Federal Studies, Vol. 25, No.4, p. 379-389. DOI: https://doi.org/10. 1080/13597566.2015.1083013 
Hickey, Sam (2012). Beyond, Poverty Reduction through Good Governance: The New Political Economy of Development in Africa. En New Political Economy, Vol.17, No.5, p. 683-90. DOI: http://dx.doi.org/10.5539/ass.v11n19p290.

\author{
Musaeva, K. M.; Zaidullaevna, A. A., y Bashirovna, A. E. (2015). Problems and \\ Ways of Improvement of Tax Instruments as a Factor in the Growth of Tax \\ Opportunities Territories. En Asian Social Science, Vol.11, No.19, p. 290-297.
}

\title{
Reflexiones Finales:
}

Durante las dos últimas décadas, las relaciones comerciales entre España y Rusia han crecido significativamente, según señala el ICEX, numerosas empresas españolas (Energía, Moda, Automoción, etc.) emprenden un proyecto de inversión en Rusia, un arduo mercado. Sin embargo, se identifica que la información existente sobre los negocios entre ambos países es todavía escasa. Puede que derivado, de la limitada faceta comercial de España como país innovador e industrializado y/o del condicionado colectivo consumidor y profesional ruso por los clásicos estereotipos. Entre los sectores más interesantes para las exportaciones españolas merecen atención los productos agroalimentarios, los bienes de consumo y el sector energético (energías renovables); en cuanto a las oportunidades de inversión, destacan los sectores de servicios sanitarios, las concesiones de carreteras y el turismo (agencia de viajes, turoperadores turísticos y hoteles). Las importaciones rusas se centran en los productos siderúrgicos, pescados y cereales.

Por otra parte, en la potencial economía rusa aún existen varios desafíos para hacer negocios, ejemplo de ello es: el aumento de los impuestos; la dependencia y necesaria relación local para la comunidad empresarial internacional que comience a invertir; la elevada burocracia y coste financiero al iniciar una empresa; las distinciones culturales; la polarización de las infraestructuras e intervención gubernamental marcada por la corrupción, el grado de monopolización y las fuertes medidas de proteccionismo. Según las previsiones, la economía rusa, dependiente del petróleo, crecerá gracias al consumo interno y el Estado acusa déficits sociales y democráticos. Mientras que el tejido empresarial español atiende, igualmente, a la dificultad en la burocracia gubernamental y las tasas impositivas. Así como, a la capacidad insuficiente de innovar y el acceso a financiamiento. Según datos recientes del Fondo Monetario Internacional (FMI), la economía española se sitúa en el puesto catorce, muy cerca de Rusia, a medio plazo se mantendrá dentro de las 20 mayores naciones por PIB. Ciertamente, esta investigación crea conocimiento, se expone que existen diferencias y similitudes en ambas economías, y que ello hace necesario profundizar en las causas. Cimienta las bases de una investigación de mayor alcance, un punto de comienzo en el fomento de un mayor discernimiento y la posibilidad de explorar las oportunidades de cooperación en el comercio bilateral. Además de mejorar la aplicación de las políticas económicas que fomenten el emprendimiento de España y Rusia. Ya en 2018, las exportaciones agroalimentarias de Andalucía a Rusia han aumentado según Extenda (Consejería de Conocimiento, Investigación y Universidad de Andalucía) y, en contraposición, Rusia es el mercado emisor de turismo hacia España que más crece desde el año pasado. Las relaciones hispano-rusas son positivas, con contactos a nivel político y económico cada vez más frecuentes. Sin duda, países con relaciones comerciales e interés compartidos que reclaman a establecer investigaciones y proyectos bilaterales. 